\title{
Uma alternativa para ensinar e aprender um processo de difusão simples usando animações no Algodoo+*
}

Samir Lacerda da Silval

Judismar Tadeu Guaitolini Junior ${ }^{2}$

Instituto Federal do Espírito Santo

Campus Vitória

Vitória - ES

Rodrigo Lacerda da Silva ${ }^{3}$

Instituto Federal Fluminense, Campus Bom Jesus do Itabapoana

Bom Jesus do Itabapoana - RJ

Emilson Ribeiro Viana Junior ${ }^{4}$

Universidade Tecnológica Federal do Paraná

Curitiba - PR

Fábio F. Leal ${ }^{5}$

Instituto Federal Fluminense, Campus Campos Centro

Campos dos Goytacazes - RJ

\section{Resumo}

Nesse trabalho, foram feitas animações no software livre Algodoo que descrevem a trajetória de uma caminhada aleatória a fim de apoiar o ensino dos conceitos de movimento Browniano. A caminhada aleatória foi modelada considerando a colisão elástica entre as partículas em suspensão num fluido. A intensidade das velocidades das partículas em nossas simulações foi definida em uma faixa arbitrária e uma orientação distribuída aleatoriamente. Usando dois métodos, o histograma de distribuição

\footnotetext{
${ }^{+}$An alternative for teaching and learning the simple diffusion process using Algodoo animations

* Recebido: novembro de 2015.

Aceito: abril de 2016.

${ }^{1}$ E-mail: samir.lacerda@ifes.edu.br; samirlsilva@gmail.com

2 E-mail: jguaitolini@ifes.edu.br

3 E-mail: rlacerda@iff.edu.br

4 E-mail: emilsonjunior@utfpr.edu.br

5 E-mail: ffleal.iff@gmail.com
} 
de deslocamentos e o deslocamento quadrático médio, foi possível medir o coeficiente de difusão para quatro sistemas diferentes, e determinar as regiões onde o sistema apresenta o regime balístico ou o difusivo. $O$ regime balístico foi observado graficamente quando o deslocamento quadrático médio tem uma dependência parabólica com o tempo, diferente do típico regime difusivo, onde o deslocamento quadrático médio tem uma dependência linear. A estratégia didática é combinara descrição matemática do movimento Browniano usando as animações com Algodoo, auxiliando os processos de ensino e aprendizagem.

Palavras-chave: Movimento Browniano; Caminhada aleatória; Coeficiente de difusão; Animação; Algodoo; Ensino de Física.

\begin{abstract}
In this work, animations of the random walk movement using a freeware Algodoo software were done in order to support the teaching of the concepts of Brownian motion. The random walk movement was simulated considering the elastic collision between the particles in suspension in a fluid. The intensity of the particles'velocities in our simulation were defined in an arbitrary range, and a random distribution of the velocity directions. Using two methods, the distribution histogram of displacements and the mean-square-displacement, it was possible to measure the diffusion coefficient of four different simulated systems, and determine the regions where the system presents ballistic regime or diffusive transport regime. The ballistic regime was observed graphically when the mean-square-displacement has a parabolic dependence with time, which differing from the typical diffusive regime where meansquare-displacement has a linear dependence. The pedagogy strategy is to blend the mathematical description of Brownian motion using animations with Algodoo, supporting the teaching and learning processes.
\end{abstract}

Keywords: Brownian motion; Random Walk; Diffusion Coefficient; Animation; Algodoo; Teaching of Physics.

\title{
I. Introdução
}

A difusão é um dos processos mais estudados e divulgados na ciência. A descrição de Einstein sobre o movimento errático de pequenas partículas na superfície de fluidos, o histórico Movimento Browniano $(\mathrm{MB})^{[1-3]}$, levou à comprovação que a matéria é constituída por átomos 
e moléculas em constante movimento em acordo com a teoria cinética. Outros numerosos sistemas exibem algum processo de difusão, como a propagação da Dengue pela trânsito de indivíduos infectados ou mosquitos ${ }^{[4]}$, a agregação por difusão limitada aplicada em fenômenos de crescimento $^{[5]}$, a análise de dados financeiros em bolsa de valores ${ }^{[6]}$, o modelo de enovelamento de proteínas ${ }^{[7]}$, o movimento de fixação ocular ${ }^{[8]}$ e muitos outros. Muitos desses processos estocásticos podem ser estudados utilizando a caminhada aleatória (random walk).

Quando esse conteúdo é ministrado em cursos tradicionais, não é fácil para o aluno obter uma compreensão intuitiva dos fenômenos estocásticos, porque isso normalmente exige ferramentas matemáticas avançadas. Atualmente, vários trabalhos vêm sendo propostos no sentido de estabelecer uma melhor abordagem pedagógica, estudando modelos simples por meio de experimentos numéricos de baixo custo que estão ao alcance de alunos com acesso a um computador $^{[9-11]}$, ou utilizando as simulações para complementar experiências reais ${ }^{[10-18]}$.

$\mathrm{O}$ uso dessas tecnologias computacionais, aliado à boas estratégias de ensino, pode fornecer ferramentas úteis para auxiliar o desenvolvimento cognitivo do estudante. E de fato, tais tecnologias vêm sendo mais utilizadas no ensino de Física nos últimos anos ${ }^{[10-18]}$. Para minimizar as dificuldades para ensinar e aprender um fenômeno físico, alguns professores têm associado às suas aulas software capazes de apresentar a evolução de equações, criar simulações e animações de um certo fenômeno, automatizar a aquisição de dados experimentais, modelar e interagir com ambientes físicos virtuais ${ }^{[10,11,18-22]}$.

No entanto, os recursos em termos de mão de obra, espaço e finanças são uma grande preocupação em muitas instituições hoje, especialmente em regiões carentes do Brasil. A escassez de recursos pode levar a turmas superlotadas e dificuldades na aquisição de kits experimentais para laboratórios ou software pagos para simulações. Uma saída seria projetar e construir ferramentas de baixo custo, que busquem minimizar o tempo de preparação pelo professor e maximizar o aprendizado dos estudantes. Neste trabalho, propomos uma ferramenta com estas características.

Estabelecemos um roteiro virtual nesse artigo para estudar e calcular um processo de difusão simples. Construímos quatro simulações de uma caminhada aleatória bidimensional para partículas na superfície de um fluido, de modo a estudar o movimento Browniano. Para realizar essa tarefa, usamos o software livre para simulação 2D chamado Algodoo da Algoryx Simulation ${ }^{[23]}$, que possui um ambiente interativo, onde é permitido criar cenários de experimentos virtuais como desenhos animados, mas tendo como feedback as equações e as propriedades físicas implementadas para as simulações. O Algodoo é uma ferramenta de fácil manipulação que não requer um conhecimento específico de programação ou treinamento para realizar as tarefas no software, permitindo uma rápida adaptação pelos alunos. Assim, mais tempo pode ser dedicado ao básico da aprendizagem através da visualização e interação com a animação ao invés de passar um longo tempo modelando o problema com linguagens de programação complicadas. Neste momento, é essencial dizer que consideramos importante o contato dos alunos com diferentes softwares, e que também aprendam, se necessário, uma linguagem de 
programação tradicional, mesmo não sendo uma tarefa fácil. Isso certamente é parte de um ciclo completo de formação de indivíduos. Nesse trabalho, no entanto, queremos fornecer uma ferramenta que evitará este caminho difícil no início, aumentando o interesse do aluno no assunto e permitindo compreender os principais conceitos físicos envolvidos e os procedimentos matemáticos para o processamento dos dados. Em um recente trabalho de nosso grupo de pesquisa $^{[24]}$, apresentamos a potencialidade do software como uma ferramenta para a física de ensino e aprendizagem, considerando as animações do lançamento de projéteis.

Acreditamos que a complementação da descrição matemática do movimento Browniano usando as animações com Algodoo irá proporcionar aos alunos uma melhor aprendizagem sobre todas as técnicas envolvidas neste modelo estocástico. Além disso, o operador pode explorar a sua criatividade investigativa, propondo modificações na animação como a adição de campos gravitacionais locais ou globais, mistura de partículas com geometrias diferentes, aumento ou diminuição do atrito, variação da elasticidade e assim por diante. Estas são as vantagens deste software no que diz respeito às numerosas ferramentas de animação que estão hoje em dia disponíveis on-line. Com este trabalho, podemos também criar a base da instrumentação teórica para o estudo de outros processos difusivos quando usamos animações do Algodoo para o movimento Browniano.

Este artigo está organizado da seguinte forma; em primeiro lugar, vamos construir as animações usando o software Algodoo e apresentar algumas das propriedades básicas da caminhada aleatória em duas dimensões para uma partícula ativa com a direção aleatória da velocidade em um ambiente homogêneo bidimensional. Por conveniência, vamos usar a imagem familiar de uma partícula de difusão. Em seguida, através das animações, vamos mostrar como calcular o coeficiente de difusão usando dois métodos: o histograma do deslocamento da partícula browniana e o deslocamento quadrático médio. Finalmente, mostramos que as animações dos passeios aleatórios fornecem uma compreensão clara da transição entre o comportamento balístico e o difusivo no movimento Browniano.

\section{O movimento Browniano e os procedimentos para construir as animações}

O sistema Browniano é constituído por uma partícula suspensa em um fluido com o movimento aleatório resultante da sua colisão com os átomos e as moléculas que constituem o fluido. Não é uma tarefa simples simular um fluido, uma vez que esta simulação envolve a solução das equações de Euler e Navier-Stokes, e assim, em nossas animações, o fluido é formado por pequenos discos azuis movendo-se aleatoriamente.

O procedimento para definir o fluido na animação consiste em criar um pequeno conjunto de discos idênticos e atribuir-lhes uma velocidade com diferentes magnitudes e direções aleatórias. Para aumentar o número de discos do fluido utilizamos o processo de copiar e colar diversas vezes um conjunto de discos original até atingir a concentração desejada. Consideramos o atrito nulo e a elasticidade máxima na colisão entre os discos para manter constante a energia cinética média do sistema. Essas características podem ser estabelecidas selecionando 
todos os discos e editando no item "Material" do software Algodoo. Confinamos as partículas em uma região retangular criando planos através da ferramenta "Plane tool". Nesse momento, iniciamos a animação pelo botão "Play" e aguardamos o fluido entrar em equilíbrio, ou seja, até a distribuição das partículas se tornar uniforme no tempo. Com a animação parada, um disco vermelho pode ser introduzido em qualquer região do sistema, representando a partícula Browniana. A partícula em suspensão (disco vermelho) é definida com um diâmetro muito maior do que as partículas do fluido (discos azuis), a fim de destacá-la das demais.

Em nossas animações estabelecemos, por conveniência, uma região plana retangular de $575.0 \mathrm{~m}^{2}$, onde foram distribuídos uniformemente $\mathrm{N}_{\mathrm{i}}$ discos azuis formando o fluido. Cada disco azul possui uma massa de $25 \mathrm{~g}$ e ocupa uma área de $12.0 \times 10^{-2} \mathrm{~m}^{2}$. Foram atribuídos módulos de velocidades entre $0.1 \mathrm{~m} / \mathrm{s}$ e $5.5 \mathrm{~m} / \mathrm{s}$ com orientações aleatórias. A partícula Browniana, isto é, o disco vermelho tem uma massa de $70 \mathrm{~g}$ e área de $3.0 \times 10^{-1} \mathrm{~m}^{2}$. Estas especificações foram escolhidas arbitrariamente e não representam qualquer material ou sistema específico. Como este software tem uma limitação para atribuir pequenas dimensões e massas aos objetos desenhados, o nosso objetivo foi criar animações fictícias da caminhada aleatória para estudar as propriedades do movimento Browniano.

Na Fig. 1(a), apresentamos a ilustração do ambiente construído para observar o movimento Browniano. Quando começamos as animações no ambiente Algodoo, a dinâmica dos discos azuis simula o movimento aleatório das moléculas em um fluido, o que pode ser visto na Fig. 1(b). Para observar a trajetória do disco vermelho, usamos a ferramenta "Tracer", que desenha uma linha através do caminho percorrido pelo objeto selecionado. Uma trajetória típica do movimento que estaremos estudando pode ser observada na Fig. 1(b) por um caminho ilustrado pela linha vermelha. Essa animação pode ser acessada através da referência ${ }^{[25]}$.

Teoricamente, os movimentos nos eixos x e y obedecem à caminhada aleatória (random walk) com o deslocamento médio bidimensional $\langle\Delta r\rangle$ nulo e o deslocamento quadrático médio (DQM) $\left\langle\Delta r^{2}\right\rangle$ dado por:

$$
\left\langle\Delta r^{2}\right\rangle=\left\langle\Delta x^{2}\right\rangle+\left\langle\Delta y^{2}\right\rangle=4 D t
$$

no qual $\left\langle\Delta x^{2}\right\rangle$ e $\left\langle\Delta y^{2}\right\rangle$ são DQM unidimensionais, $D$ é o coeficiente de difusão e $t$ o tempo entre os pontos dos dados ${ }^{[1-3,26,27]}$. Assim, o DQM depende do coeficiente de difusão e do tempo. $\mathrm{O}$ deslocamento quadrático médio (root mean square $-r m s$ ) $\left\langle\Delta r^{2}\right\rangle_{r m s}=\sqrt{\left\langle\Delta r^{2}\right\rangle}$ é proporcional a $\sqrt{t}$, permitindo que o disco vermelho visite rapidamente a área vizinha do ponto de lançamento e que necessite de um tempo grande para atingir distâncias longas ${ }^{[27]}$. 


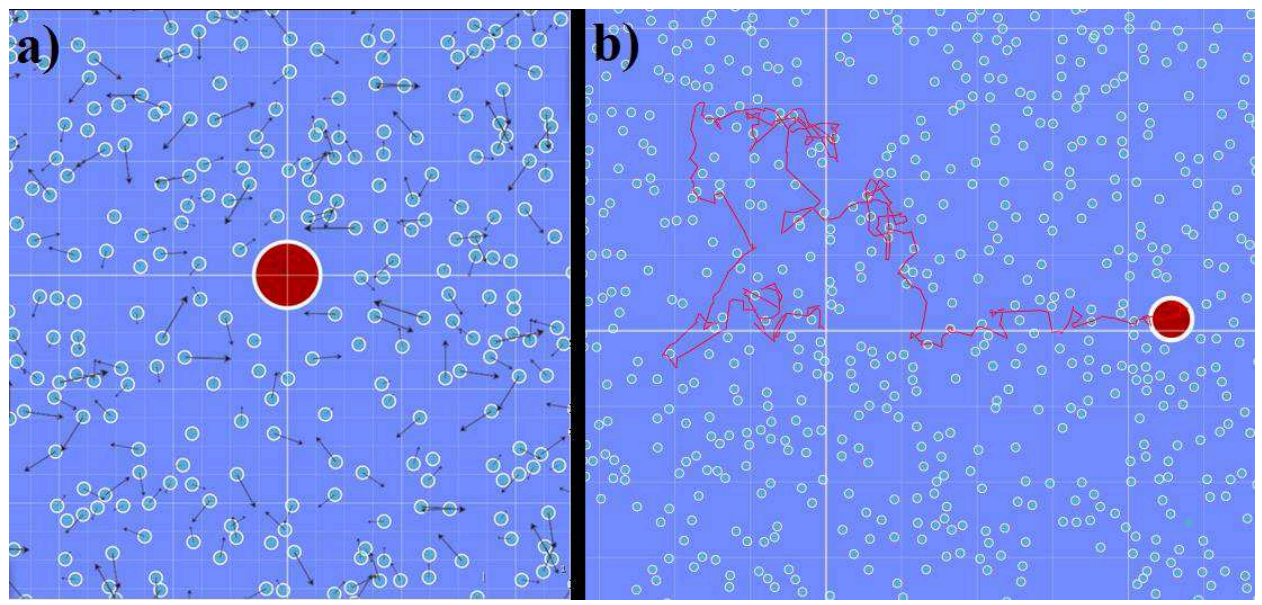

Fig. 1 - Ilustração da animação do movimento Browniano. Os pequenos discos representam o fluido e o disco maior é a partícula Browniana que vai executar o movimento aleatório devido a colisões entre as partículas do fluido.

O movimento Browniano apresenta uma transição entre o comportamento balístico suave e o comportamento difusivo. A escala de tempo da transição é dada pelo tempo de relaxação $\tau$. Em sistemas físicos reais, o tempo de relaxação $\tau$ é geralmente muito pequeno, tipicamente na ordem de alguns microssegundos ou nanossegundos ${ }^{[27-30]}$. Para um intervalo de tempo curto $\Delta t\left\langle\tau,\left\langle\Delta r^{2}\right\rangle\right.$ tem uma dependência quadrática no tempo, e para tempos longos $\left.(\Delta t\rangle \tau\right)$ essa dependência torna-se linear ${ }^{[27-30]}$. Embora a teoria tenha sido proposta em $1905^{[2]}$, a transição entre o comportamento balístico e difusivo foi experimentalmente provado apenas em 2010 por Li, T., Kheifets ${ }^{[30]}$, quando foi possível medir a posição da partícula para estudar a velocidade instantânea e a transição do regime balístico ao difusivo.

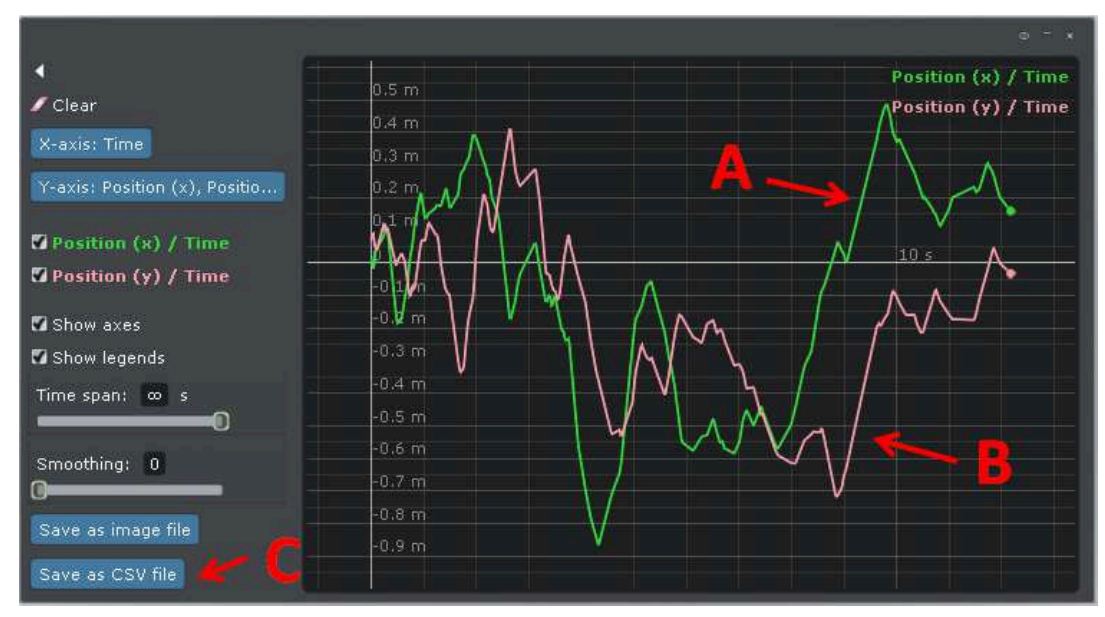

Fig. 2 - Gráfico no Algodoo apresentando a série temporal da posição x e y para o disco Browniano. 
Para estudar o movimento Browniano pela animação do Algodoo utilizamos a ferramenta gráfica "Show Plot", com a qual podemos escolher algumas variáveis envolvidas na animação e apresentá-las em um gráfico, como ilustrada na Fig. 2. No caso do movimento Browniano, as evoluções temporais das posições horizontal $x(t)$ e vertical $y(t)$ do disco Browniano foram tratadas como séries temporais. A opção "Show Plot" exibe as posições x e y do disco em função do tempo, que podem ser observadas na Fig. 2 nos itens (A) e (B) respectivamente e com esta ferramenta foi possível ainda salvar uma planilha na extensão .CSV (item (C) da Fig. 2) com uma frequência de 60 pontos por segundo. Dado que cada amostra apresenta uma condição inicial diferente, os arquivos CSV com as séries temporais da posição do disco Browniano foram usados para analisar o movimento.

Em nossas animações, o coeficiente de difusão $D$ pode ser obtido por dois métodos. O primeiro método baseia-se na avaliação do gráfico da distribuição de probabilidade da posição de partícula. Usando este gráfico, podemos ajustar a curva Gaussiana usando

$$
P(x)=\frac{1}{\sqrt{2 \pi \sigma^{2}}} e^{-\frac{x^{2}}{2 \sigma^{2}}}
$$

com a variância $\sigma^{2}$ no movimento Browniano igual ao $\mathrm{DQM}\left\langle\Delta x^{2}\right\rangle$, já que o deslocamento médio $\langle\Delta x\rangle$ é nulo nesse caso.

$$
\sigma^{2}=\left\langle\Delta x^{2}\right\rangle-\langle\Delta x\rangle^{2}
$$

Podemos usar a variância $\sigma^{2}$ da distribuição de deslocamentos $\left\langle\Delta x^{2}\right\rangle$ para calcular $D$ pela equação da caminhada aleatória unidimensional

$$
\sigma^{2}=\left\langle\Delta x^{2}\right\rangle=2 D t
$$

Usando qualquer software que analise planilhas matematicamente, como o Microsoft Excell $^{\circledR}$, Open Office ${ }^{\circledR}$ ou o Origin ${ }^{\circledR}$, podemos fazer o tratamento matemático das séries temporais. Calculando os deslocamentos horizontais e verticais $\Delta x_{i}=x_{i}(t)-x_{i}\left(t_{0}\right)$ e $\Delta y_{i}=y_{i}(t)-$ $y_{i}\left(t_{0}\right)$ é possível criar uma lista e produzir o gráfico de distribuição de probabilidade da posição da partícula, no qual será ajustada a equação 3.

O segundo método consiste em calcular o DQM bidimensional para cada instante de tempo $t$ usando a equação

$$
\left\langle\Delta r^{2}(t)\right\rangle=\frac{1}{N} \sum_{i=1}^{N}\left[\left(x_{i}(t)-x_{i}\left(t_{0}\right)\right)^{2}+\left(y_{i}(t)-y_{i}\left(t_{0}\right)\right)^{2}\right]
$$

na qual o par cartesiano $\left(x_{i}\left(t_{0}\right), y_{i}\left(t_{0}\right)\right)$ representa a posição inicial da partícula em cada amostra e $N$ é o número de amostras. O coeficiente de difusão $D$ é a inclinação do ajuste linear da curva $\left\langle\Delta r^{2}(t)\right\rangle$ versus $t$, de acordo com a equação 1 .

Para demonstrar a transição entre o comportamento balístico e difusivo, utilizamos a curva do DQM para um intervalo de tempo curto gerado pelo segundo método. A partir do gráfico de $\left\langle\Delta r^{2}(t)\right\rangle$ versus $t$, podemos demonstrar o comportamento temporal quadrático e linear de $\left\langle\Delta r^{2}(t)\right\rangle$ e, a partir desse gráfico, obter o tempo de relaxação $\tau$. 
Nas aulas de laboratório para alunos de graduação, um experimento típico de movimento Browniano exige que o aluno registre o movimento de pelo menos dez partículas para medir o coeficiente de difusão e/ou o tempo de relaxação. Experimentos do movimento Browniano em estágios avançados tornaram-se um marco, porém o custo de preparação e a quantificação das experiências ainda são difíceis, geralmente produzindo desvios de $10 \%$ a $15 \%$ ou mais $^{[26]}$.

Em termos práticos, o estudo do movimento Browniano através de experimentos computacionais minimiza grande parte dos problemas experimentais, preparando o aluno mais rapidamente para o estudo de processos de difusão utilizando as técnicas matemáticas e análise gráfica. Nesse cenário, o software Algodoo é uma boa opção, se destacando por possuir uma fácil manipulação e não requerer um conhecimento específico de programação. Não propomos aqui a substituição de experimentos por simulações computacionais, mas apresentamos uma nova ferramenta de apoio ao ensino/aprendizagem de física através de animações no Algodoo. Desta forma, quando o aluno tiver contato com experiências reais que envolvem o movimento Browniano, já terá o conhecimento das quantidades físicas a serem coletadas e o tratamento matemático necessário para obter as propriedades do sistema como, por exemplo, o coeficiente de difusão.

\section{Resultados e discussões}

Com as animações em execução, os professores têm uma grande oportunidade para discutir com seus alunos sobre a agitação dos pequenos discos e associá-los qualitativamente à agitação térmica do fluido hipotético. Essa agitação térmica produz constantes choques com o disco vermelho localizado nessa região, causando um movimento errático, caracterizando o movimento Browniano (Fig. 1 (a) e (b)). Devido ao fato deste software ser facilmente manipulado, o professor pode interromper, aumentar ou diminuir a velocidade da animação, ou também modificar a característica do ambiente para enriquecer a apresentação qualitativa do movimento.

Como dito na seção anterior, a série temporal das posições do disco browniano foi salva pela ferramenta "Show Plot", em uma extensão .CSV e os dados tratados e analisados em um programa de planilha. Construímos quatro animações independentes com $N_{i}$ discos azuis formando o fluido do sistema, $\operatorname{com} i=1,2,3$ e 4 , onde $N_{1}=3600, N_{2}=5400, N_{3}=7200$ e $N_{4}=$ 9000 partículas, e identificamos os sistemas por $S_{1}, S_{2}, S_{3}$ e $S_{4}$. Executamos para cada sistema duzentos movimentos Brownianos independentemente durante o intervalo de $15 \mathrm{~s}$, sendo que para cada animação, $S_{i}$ irá produzir um coeficiente de difusão $D_{i}$.

Ao aplicar o método \#1 para cada sistema $S_{i}$, ajustamos uma curva Gaussiana (equação 3) para o gráfico de distribuição de deslocamento na Figura 3. O histograma é construído a partir dos deslocamentos independentes unidimensionais $\Delta x$ e $\Delta y$ juntos. A variância da curva Gaussiana ajustada é igual ao valor do deslocamento quadrático médio unidimensional $\left\langle\Delta r^{2}\right\rangle$, e podemos usar isso para calcular o coeficiente de difusão $D_{i}$ usando a equação 5 . 


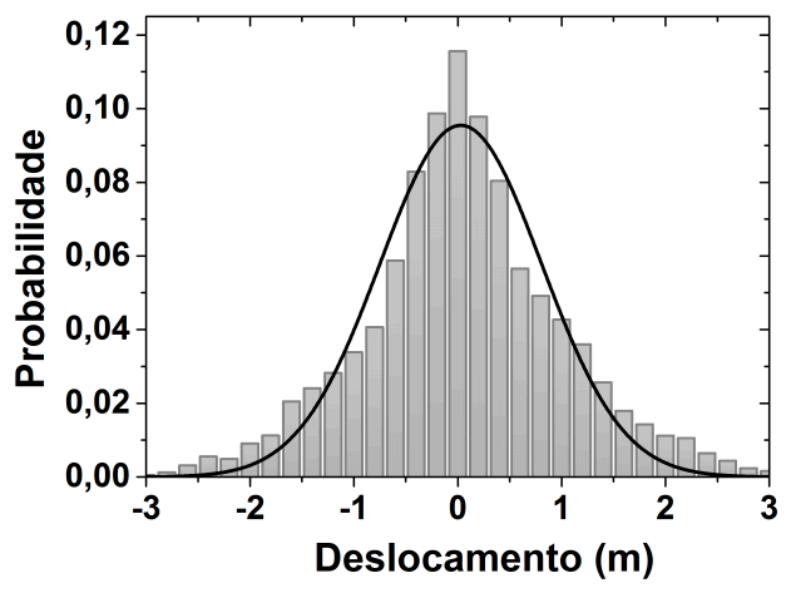

Fig. 3 - Pelo método \#1, o coeficiente de difusão é encontrado fazendo um histograma dos deslocamentos em x e y (cinza) e o ajuste de uma curva Gaussiana (linha preta) para os dados; a variância é usada para calcular o coeficiente de difusão de $D_{i}$. A figura foi calculada para $\mathrm{N}_{2}=5400$ partículas.

Tabela 1. O coeficiente de difusão Di calculado pelo método \#1 para cada sistema Si com diferentes intervalos de tempo.

\begin{tabular}{lllll}
$D_{i}\left[\mathrm{~m}^{2} / s\right]$ & $\Delta t_{1}=6,7 \mathrm{~s}$ & $\Delta t_{2}=7,5 \mathrm{~s}$ & $\Delta t_{3}=8,5 \mathrm{~s}$ & $\Delta t_{4}=10,0 \mathrm{~s}$ \\
$\mathrm{D}_{1}$ & 0,276 & 0,324 & 0,373 & 0,473 \\
$\mathrm{D}_{2}$ & 0,158 & 0,185 & 0,209 & 0,256 \\
$\mathrm{D}_{3}$ & 0,103 & 0,126 & 0,149 & 0,189 \\
$\mathrm{D}_{4}$ & 0,141 & 0,166 & 0,190 & 0,237 \\
\hline
\end{tabular}

Na Tabela 1, é apresentado o coeficiente de difusão $D_{i}$ calculado para cada $S_{i}$ em diferentes intervalos de tempo. Na medida em que analisamos o sistema para intervalos de tempo maiores, a dispersão das partículas aumenta, alterando a variância $\sigma^{2}$, e consequentemente, $D_{i}$. A Fig. 4 mostra o comportamento de coeficiente de difusão para cada sistema $S_{i}$ de acordo com o intervalo de tempo. Para um sistema com um número fixo de partículas, $D_{i}$ cresce à medida que o intervalo de tempo aumenta, mas para um intervalo de tempo fixo, $D_{i}$ é maior para os sistemas com menor número de partículas.

Após determinar o deslocamento quadrático da posição para todos os discos Brownianos (Fig. 5 - curvas cinza), calculamos a média sobre todas as amostras, isto é, o DQM (Fig. 5 - curva preta) pela equação 5 . O coeficiente de difusão foi determinado pela inclinação do DQM versus o tempo utilizando a equação 1 , onde obtemos $D_{i}$ utilizando o método \#2 para cada sistema. 


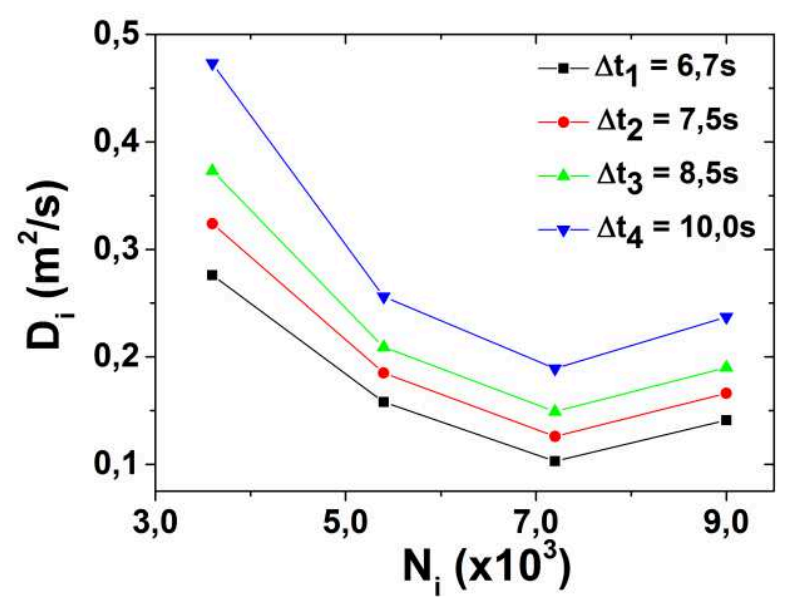

Fig. 4 - Coeficiente de difusão Di versus número de partículas Ni para cada intervalo de tempo $\Delta t_{i}$, com $i=1,2,3$ e 4 .

Tabela 2. O coeficiente de difusão Di calculada para cada Ni em diferentes intervalos de tempo pelo método \#2.

\begin{tabular}{lllll} 
Unit & $D_{1}$ & $D_{2}$ & $D_{3}$ & $D_{4}$ \\
$\mathrm{~m}^{2} / \mathrm{s}$ & 0.27 & 0.32 & 0.37 & 0.4 \\
& 6 & 4 & 3 & 73 \\
\hline
\end{tabular}

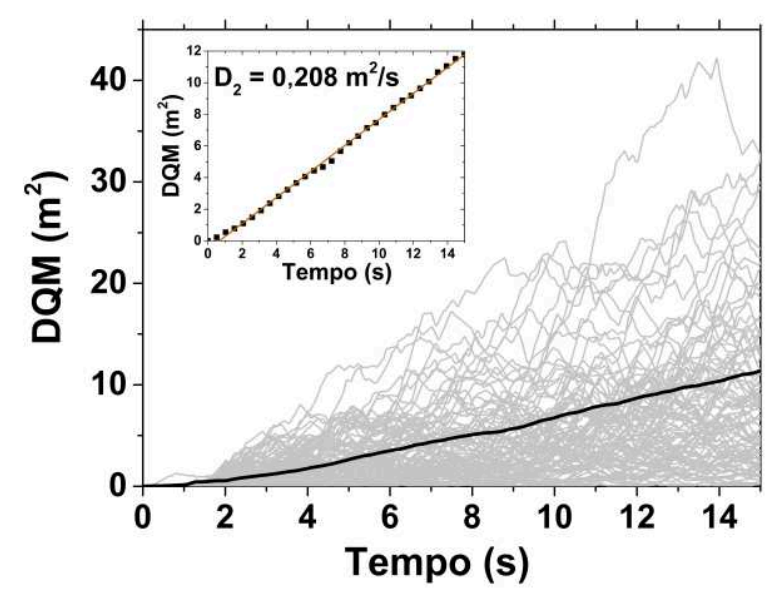

Fig. 5 -Deslocamento quadrático de 200 amostras para $N_{2}=5400$ partículas (curvas em cinza) e deslocamento quadrático médio em função do tempo (curva em preto). Em detalhe, o ajuste linear para DQM com $D_{2}=0,208 \mathrm{~m}^{2} / \mathrm{s}$.

Agora, na Tabela 2, são apresentados os coeficientes de difusão $D_{i}$ calculados para cada sistema pelo o método \#2, e uma mudança no coeficiente de difusão pode ser observada em função de $N_{i}$. O coeficiente de difusão diminui à medida que o número de partículas do sistema aumenta. Os coeficientes de difusão obtidos a partir do método \#2 (Tabela 2) são semelhantes aos obtidos a partir do método \#1 (Tabela 1), porém mais precisos. Na Fig. 6 são 
apresentados os coeficientes de difusão obtidos pelos métodos \#1 e \#2 em função do número de partículas. Nesse caso, selecionamos os $D_{i}$ do método \#1 que melhor ajustam aos pontos obtidos pelo método \#2.

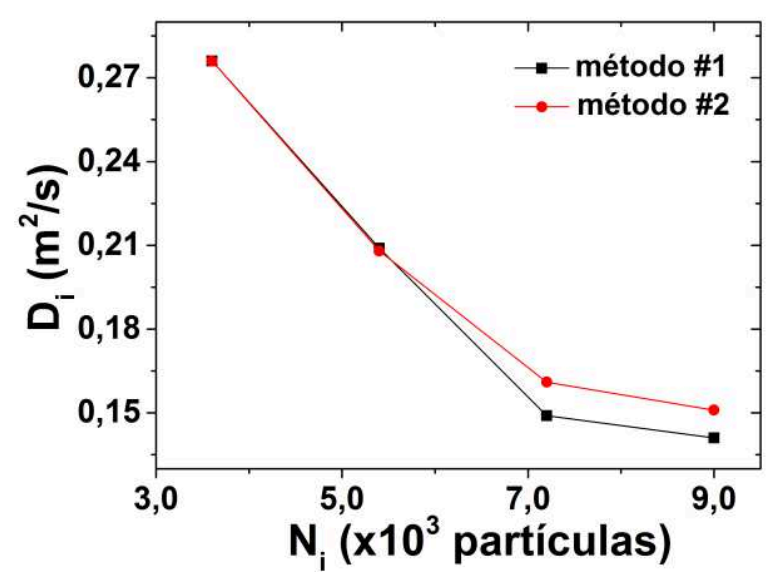

Fig. 6 - Comparação entre o método \#1 (preto) e o método \#2 (vermelho) do coeficiente de difusão $D_{i}$ versus o número de partículas de $N_{i}$.

Para um intervalo de tempo curto $(\Delta t<\tau)$ a dinâmica do disco vermelho Browniano é governada pela sua inércia e o movimento é balístico. A Fig. 7 mostra que DQM tem uma dependência parabólica com o tempo (curva com pontos vermelhos), que difere do típico movimento difusivo (pontos pretos), onde DQM tem uma dependência linear. Avaliamos o tempo de relaxação $\tau_{i}$ para cada sistema $S_{i}$ e eles são apresentados na Tabela 3, de onde observamos que o tempo de relaxação $\tau_{i}$ diminui quando o número de partículas do sistema aumenta. $\mathrm{Na}$ região parabólica do DQM da Fig. 7, ajustamos uma função polinomial de segundo grau $\left\langle\Delta r^{2}\right\rangle(t)=C t^{2}+B t+A$, cujos coeficientes $C_{i}, B_{i}$ e $A_{i}$ foram obtidos para cada sistema $S_{i}$.

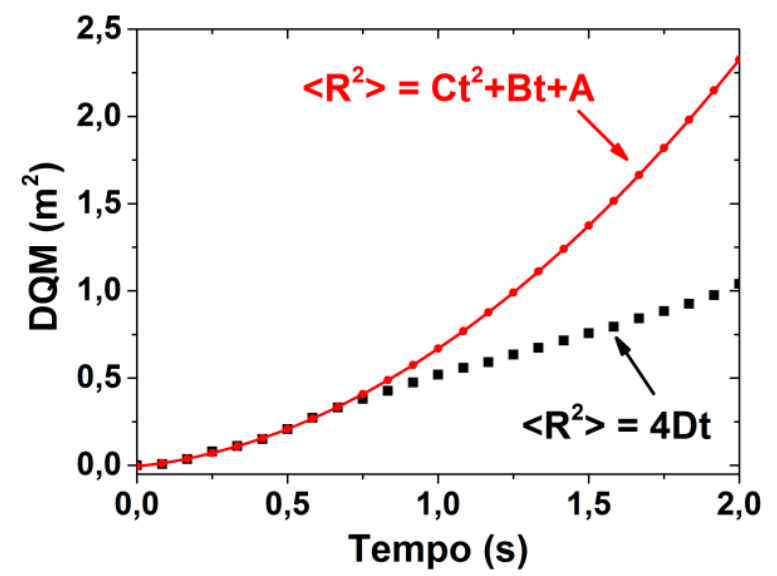

Fig. 7 - DQM é balístico para $(\Delta t<\tau) \operatorname{com}\left\langle\Delta r^{2}\right\rangle \propto t^{2}-$ Ajuste (curva vermelha) $\operatorname{com}\left\langle\Delta r^{2}\right\rangle(t)=C t^{2}+B t+A$. (Preto) Para tempo longos $(\Delta t \gg \tau)\left\langle\Delta r^{2}\right\rangle$ torna-se linear. $A$ figura foi calculada para $N_{2}=5400$ partículas. 
Tabela 3. O tempo de relaxação $\tau$ i avaliado para cada sistema Si e ajuste de parâmetros.

\begin{tabular}{ll|lll} 
& {$[\mathrm{s}]$} & $C_{i}\left[\mathrm{~m}^{2} / \mathrm{s}^{2}\right]$ & $B_{i}\left[\mathrm{~m}^{2} / \mathrm{s}\right]$ & $A_{i}\left[\mathrm{~m}^{2}\right]$ \\
$\tau_{1}$ & 1,12 & $1,46 \times 10^{-3}$ & $1,73 \times 10^{-1}$ & 5,14 \\
& & & & $\times 10^{-1}$ \\
$\tau_{2}$ & 0,70 & $-5,94 \times 10^{-3}$ & $1,84 \times 10^{-1}$ & 4,91 \\
& & & & $\times 10^{-1}$ \\
$\tau_{3}$ & 0,30 & $-7,77 \times 10^{-4}$ & $3,71 \times 10^{-2}$ & 9,41 \\
& & & & $\times 10^{-1}$ \\
& & & & \\
$\tau_{4}$ & 0,28 & $4,71 \times 10^{-4}$ & $2,51 \times 10^{-3}$ & 8,16 \\
& & & & $\times 10^{-1}$ \\
\hline
\end{tabular}

Estes resultados são apenas um pequeno exemplo do que pode ser feito usando o software Algodoo. Alterando a animação do movimento Browniano seria possível gerar outros regimes de difusão, que podem ser usados para ensinar outros fenômenos difusivos, como a difusão elétrica em materiais semicondutores ou o processo de osmose ${ }^{[15]}$.

Essa animação pode ser utilizada para vários níveis de ensino. Para níveis de ensino básico, a animação do movimento Browniano pode ser utilizada para ilustrar o movimento aleatório de uma partícula em suspensão em um fluido, dando foco aos conceitos físicos e análise qualitativa, sem o rigor matemático, análise de dados e tabelas. Para o nível de graduação, a animação pode ser uma ferramenta que facilite também a compreensão dos procedimentos matemáticos envolvidos no estudo do movimento Browniano. A partir dos dados coletados pelo Algodoo, o professor pode elaborar roteiros para ensinar os alunos como obter as quantidades físicas da série temporal, da mesma forma como é feito em experimentos de laboratório. Além disso, seria uma introdução interessante aos conceitos de física estatística que são importantes em diferentes áreas da física e química.

Em níveis mais avançados, os alunos podem aprender procedimentos científicos utilizados para formulação de modelos, coleta e tratamento de dados. Esse conhecimento básico pode vir a auxiliar o aluno, que está começando no mundo da ciência, a atuar em um projeto de pesquisa.

\section{Conclusões}

Neste artigo, mostramos uma ferramenta computacional de grande potencial para o ensino e aprendizagem de física, que são as animações feitas no software livre Algodoo. Utilizando uma animação baseada no movimento da caminhada aleatória bidimensional de partículas sobre a superfície do fluido foi possível abordar os conceitos básicos do movimento Browniano. 
As animações apresentadas neste trabalho fornecem aos professores e alunos uma ferramenta simples para análises qualitativas e quantitativas do movimento Browniano, com base na física estatística. É possível observar e discutir a caminhada aleatória dos pequenos discos produzindo um movimento irregular, e associar qualitativamente à excitação térmica de um fluido hipotético, caracterizando o movimento Browniano.

O coeficiente de difusão foi calculado a partir de quatro animações por dois métodos distintos: Método \#1 - ajuste de uma curva gaussiana em um gráfico de distribuição de deslocamentos independentes unidimensionais $\Delta x$ e $\Delta y$ juntos. Método \#2 - por um ajuste no gráfico DQM em função do tempo, utilizando a equação 1. Em ambos os casos, o coeficiente de difusão decresce à medida que o número de partículas do sistema aumenta.

Demonstramos ainda que para um tempo curto $(\Delta t<\tau),\left\langle\Delta r^{2}(t)\right\rangle$ é proporcional a $t^{2}$ e o movimento apresenta um comportamento balístico, onde temos o movimento das partículas sem sofrer colisões no caminho. Para tempos longos $(\Delta t\rangle \tau),\left\langle\Delta r^{2}(t)\right\rangle$ é proporcional a $t$ e o movimento é difusivo, sofrendo colisões ao longo do caminho. O tempo de relaxamento diminui à medida que o número de partículas aumenta.

Através do ambiente virtual utilizado, educadores e alunos podem explorar outras potencialidades do tema estudado, propondo modificações no tamanho das partículas e na intensidade das velocidades, além de poderem impor condições de contorno para o sistema e discutir suas consequências para melhorar o tempo de relaxamento, redução o coeficiente de difusão e assim por diante.

Muitos sistemas de difusão podem ser explorados por esta proposta inicial de uma animação para o movimento Browniano. A estratégia didática é combinar a descrição matemática do movimento Browniano com as animações com Algodoo, buscando um melhor processo de ensino e aprendizagem. Desta forma, Algodoo também é um instrumento de apoio que torna o processo de ensino e a aprendizagem de física mais simples e proveitosa quando comparados com outros software de animações e de aprendizagem ${ }^{[9-22]}$.

\section{Referências}

[1] SALINAS, S. R. A. Einstein e a teoria do movimento browniano. Revista Brasileira de Ensino de Física, v. 27, p. 263-269, 2005.

[2] EINSTEIN, A. On the movement of small particles suspended in stationary liquids required by the molecular-kinetic theory of heat. Annals of Physics, Berlin, v. 15, p. 549, 1905.

${ }^{[3]}$ SILVA, J. M.; LIMA, J. A. S. Quatro abordagens para o movimento browniano. Revista Brasileira de Ensino de Física, v. 29, p. 25-35, 2007.

${ }^{[4]}$ SILVA, S. L.; FERREIRA, J. A.; MARTINS, M. L. Epidemic spreading in a scale-free network of regular lattices. Physica A, v. 377, p. 689-697, 2007. 
${ }^{[5]}$ BRAGA, F. L.; RIBEIRO, M. S. Diffusion Limited Aggregation: Algorithm optimization revisited. Computer Physics Communications, v. 188, p. 1602-1605, 2011.

${ }^{[6]}$ NAKAMURA, T.; SMALL, M. Tests of the random walk hypothesis for financial data. Physica A, v. 377, p. 599-615, 2007.

${ }^{[7]}$ HUANG, K. Conditioned self-avoiding walk (CSAW): stochastic approach to protein folding. Biophysical Reviews and Letters, v. 2, p. 139-154, 2007.

${ }^{[8]}$ MAKARAVA, N.; BETTENBUHL, M.; ENGBERT, R.; HOLSCHNEIDER, M. Bayesian estimation of the scaling parameter of fixational eye movements. Europhysics Letters, v. 100, p. 4003, 2012.

${ }^{[9]}$ FIGUEIRA, J. S. Movimento browniano: uma proposta do uso das novas tecnologias no ensino de física. Revista Brasileira de Ensino de Física, v. 33, p. 4403, 2011.

[10] ZACHARIADOU, K.; YIASEMIDES, K.; TROUGKAKOS, N. A low-cost computercontrolled Arduino-based educational laboratory system for teaching the fundamentals of photovoltaic cells. European Journal of Physics, v. 33, p. 1599-1610, 2012.

[11] MURADOGLU, M.; WEI NG, E. M.; WAH NG, T. Experimentation on recurrent sphere collision with Audacity. European Journal of Physics, 35, 065017. (2014)

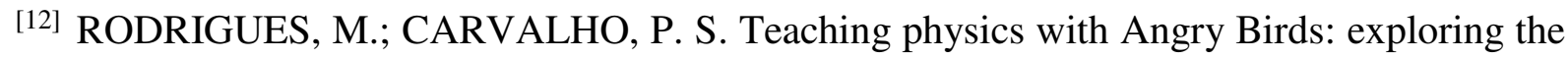
kinematics and dynamics of the game. Physics Education, 48, 431-437. (2013)

${ }^{[13]}$ HURKALA, J.; GALL, M.; KUTNER, R.; MACIEJCZYK, M. Real-time numerical simulation of the Carnot cycle. European Journal of Physics, v. 26, p. 673-680, 2005.

[14] WEE, L. K. One-dimensional collision carts computer model and its design ideas for productive experiential learning. Physics Education, v. 47, p. 301-308, 2012.

${ }^{[15]}$ KOTTONAU, J. An Interactive computer model for improved student understanding of random particle motion and osmosis. Journal of Chemical Education, 88, 772-775. (2011)

${ }^{[16]}$ Matsumoto, P. S. Exploring interactive and dynamic simulations using a computer algebra system in an advanced placement chemistry course. Journal of Chemical Education, v. 91, p. 1326-1333, 2014.

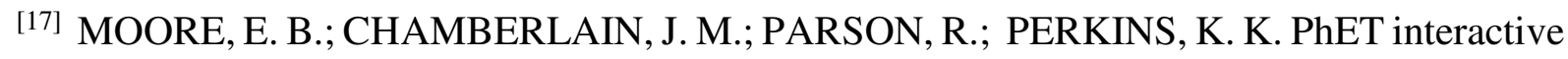
simulations: transformative tools for teaching chemistry Journal of Chemical Education, v. 91, n. 8, p. 1191-1197, 2014.

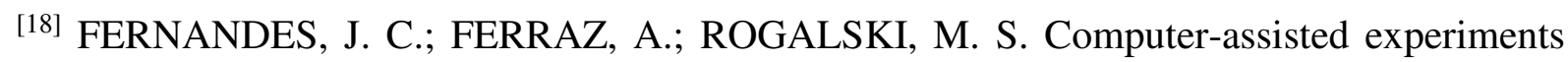
with oscillatory circuits. European Journal of Physics, v. 31, p. 299-306, 2010. 
[19] GARCIA-MARCH, M. A.; CÓRDOBA, P. F.; URCHEGUÍA, J. F.; MONSORIU, J. A. Introductory quantum physics courses using a LabVIEW multimedia module. Computer Applications in Engineering Education, v. 15, p. 124-133, 2007.

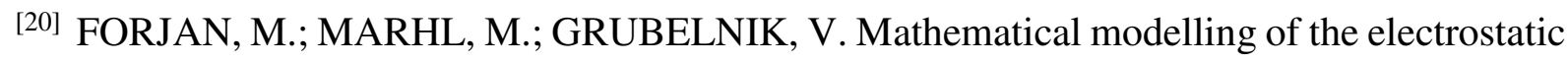
pendulum in school and undergraduate education. European Journal of Physics, v. 28, p. $015022,2014$.

[21] TEODORO, V. D.; NEVES, R. G. Mathematical modelling in science and mathematics education. Computer Physics Communications, v. 182, p. 8-10, 2011.

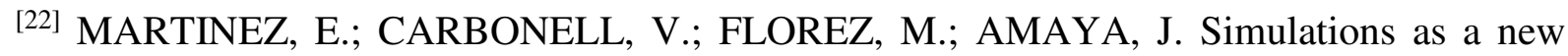
physics teaching tool. Computer Applications in Engineering Education, v. 18, p. 757-761, 2010.

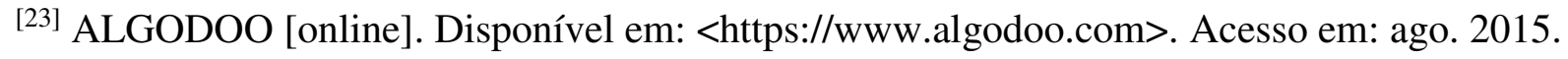

${ }^{[24]}$ DA SILVA, S. L.; DA SILVA, R. L.; GUAITOLINI JUNIOR, J. T.; GONÇALVES, E.; VIANA, E. R.; WYATT, J. B. L. Animation with Algodoo: a simple tool for teaching and learning physics. Exatas Online, v. 5, p. 28-39, 2014.

[25] Animação do processo de difusão simples [online]. Disponível em: <http://www.algodoo.com/algobox/details.php?id=129617 > . Acesso em: abr. 2016.

[26] CATIPOVIC, M. A.; TYLER, P. M.; TRAPANI, J. G.; CARTERA, A. R. Improving the quantification of Brownian motion. American Journal of Physics, v. 81, p. 485-491, 2013.

[27] VOLPE, G.; VOLPE, G. Simulation of a Brownian particle in an optical trap. American Journal of Physics, v. 81, p. 224, 2013.

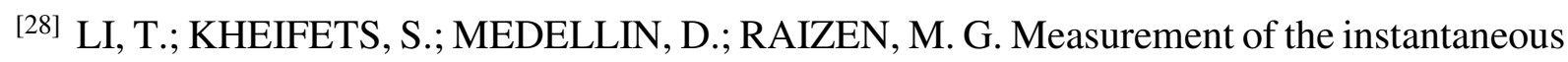
velocity of a Brownian particle. Science, v. 328, p. 1673-1675, 2010.

${ }^{\text {[29] }}$ LI, T.; RAIZEN, M. G. Brownian motion at short time scales. Annals of Physics, v. 525, p. 281-295, 2013.

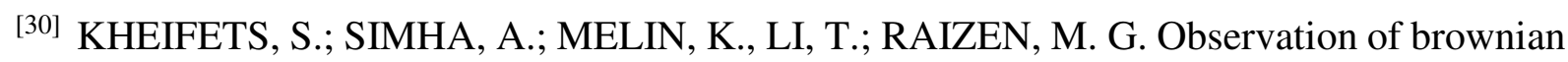
motion in liquids at short times. Science, v. 343, p. 1493, 2014. 